\title{
El giro a la derecha de la política Suramericana y sus implicaciones en la integración económica regional
}

\section{The right turn of South american policy and its implications on regional economic integration}

\section{Marco Antonio Burgos Flórez}

Magister en Política Económica Internacional, Universidad de Belgrano, Buenos Aires - Argentina, Especialista en Gerencia social, Especialista en Docencia Universitaria, Especialista en finanzas Universidad de Nariño. Docente Tiempo Completo del Departamento de Economía, Universidad de Nariño. Director Grupo de Investigación Coyuntura Económica y Social CES, Universidad de Nariño. Docente Postgrados Universidad de Nariño. Email: marcoantonioburgos@gmail.com

Doi: https://doi.org/10.22267/rceilat.204647.91

\section{Resumen}

El giro dado por Argentina y Brasil hacía gobiernos de derecha, establecen un nuevo escenario para la integración regional, en donde el cambio por una economía más liberal, con perfiles más hegemónicos y menos críticas a la integración regional más profunda y en ámbitos de ampliación del comercio a contextos continentales, le genera un nuevo espacio a profundizar las relaciones con las economías que giran en ámbitos de relaciones hegemónicas y que han tenido un marcado regionalismo abierto como Colombia, México, Chile y Perú.

La crisis del socialismo del Siglo XXI, pone de relieve la pérdida de los espacios para los argumentos anti hegemónicos y las críticas a las relaciones extra latinoamericanas, lo cual aunado a las políticas revisionistas del gobierno norteamericano, generan como lo han manifestado la Comisión Económica para América Latina CEPAL, el Banco Interamericano de desarrollo BID y otros analistas, un nuevo escenario para avanzar en la integración regional, en apoyo a un regionalismo abierto.

Palabras Clave: Giro a la derecha; Integración; Regionalismo; Proteccionismo. 


\begin{abstract}
The turn taken by Argentina and Brazil towards right-wing governments establishes a new scenario for regional integration, where the change for a more liberal economy, with more hegemonic profiles and less critical to deeper regional integration and in areas of expansion of the trade in continental contexts, generates a new space to deepen relations with the economies that revolve in areas of hegemonic relations and that have had a marked open regionalism such as Colombia, Mexico, Chile and Peru.

The crisis of socialism of the 21st century, highlights the loss of spaces for anti-hegemonic arguments and criticisms of extra-Latin American relations, which, together with the revisionist policies of the US government, generate as the Economic Commission has stated for Latin America CEPAL, the Inter-American Development Bank BID and other analysts, a new scenario to advance regional integration, in support of open regionalism.
\end{abstract}

Keywords: Right turn; Integration; Regionalism; Protectionism.

\section{Introducción}

En una aproximación a la definición de la derecha, el politólogo holandés C. Mudde (2007), establece que "la derecha puede ser definida como un grupo-partido-movimiento que estima que, la desigualdad existente es el reflejo de un proceso natural y, por consiguiente, no debe ser regulado directamente por el Estado" (Citado en Jeifets \& Jeifets, 2016, p.38). Aspecto que está en concordancia con lo expresado por Bobbio (1996) al determinar que "El alma de la derecha puede ser expresada sintéticamente con el lema: «Nada fuera ni en contra de la tradición, todo en y por la tradición»”. A lo cual el mismo autor en una explicación más amplia, establece que "el hombre de derecha es el que se preocupa, ante todo, de salvaguardar la tradición; el hombre de izquierda, en cambio, es el que entiende, por en- cima de cualquier cosa, liberar a sus semejantes de las cadenas que les han sido impuestas por los privilegios de raza, de casta, de clase, etcétera" (Bobbio, 1996, p. 116). Ello induce a afirmar que casi todos los actuales gobiernos en Suramérica (con excepción de Venezuela y Bolivia) son de derecha, dándose un giro político en la región, cuando hasta mediados de la presente década la mayoría de países eran regidos por gobiernos de izquierda (con excepción de Colombia y Perú), denominándose a dicho cambio un giro a la derecha de la región.

Ese giro a la derecha, en especial el dado por Argentina y Brasil, establecen un nuevo escenario para la integración regional, en donde el cambio por una economía más liberal, con perfiles más hegemónicos y menos críticas a la integración regional más profunda y en ámbitos de ampliación 
del comercio a contextos continentales, le genera un nuevo espacio a profundizar las relaciones con las economías que giran en ámbitos de relaciones hegemónicas y que han tenido un marcado regionalismo abierto como Colombia, México, Chile y Perú.

La crisis del socialismo del Siglo XXI, pone de relieve la pérdida de los espacios para los argumentos anti hegemónicos y las críticas a las relaciones extra latinoamericanas, generan como lo han manifestado la Comisión Económica para América Latina CEPAL y el Banco Interamericano de desarrollo BID, un nuevo escenario para avanzar en la integración regional.

\section{El giro a la derecha en Suramérica}

En primer lugar, se puede establecer que no se hará alusión a los impactos en el ámbito económico y social de dicho giro, sino que se tratará de mirar cómo esos nuevos ámbitos de una nueva política más soportada en el mercado, vislumbra un nuevo futuro para la integración regional suramericana.

En lo que se podría afirmar como el giro a la derecha en el Mercosur, se destacan los grandes cambios en los gobiernos de Brasil, Argentina, Paraguay y en menor medida del Uruguay. Donde el hito que ha marcado el giro a la derecha suramericana lo constituye sin lugar a dudas, la elección del presidente brasilero Jair Bolsonaro (2018-2022), con la transición de dos gobiernos de izquierda como el de Lula da Silva (2003-2010) y Dilma
Rousseff (2011-2016), que han dirigido el país desde el año 2003. El analista Mauricio Jaramillo Jassir (2018), comenta que Bolsonaro en torno a su posición regional: "Ha hablado de salirse de Mercosur y contempla la posibilidad de salirse de Unasur. Brasil siempre había sido antiimperialista y él se quiere acercar a Estados Unidos. Con esto, se aislaría de asuntos regionales y habría un retroceso" (Citado en Gil y Flórez, 2018, p 1).

El avance de la derecha en Argentina con el presidente Mauricio Macri (2015-2019), es rotundo, siendo el remplazo del gobierno de la presidenta Cristina Fernández de Kirchner, cuyo partido estuvo al frente del gobierno en dos ocasiones (2007-2015).

También en el ámbito del Mercosur para el año 2017, se destaca el cambio de Gobierno en Paraguay del presidente Lugo por el presidente "conservador Mario Abdo Benítez, hijo del secretario particular del dictador Alfredo Stroessner" (Riesco, 2018).

En Uruguay en el año 2015, el expresidente Tabaré Vásquez gana la presidencia, en remplazo de José $\mathrm{Mu}-$ jica (2010-2015), cuya primera presidencia fue a nombre del Partido socialista y la segunda con "el Frente Amplio y con propuestas más de centro izquierda” (Riesco, 2018).

En el otro lado del continente, se destaca el giro a la derecha de los países de Ecuador y Chile. Siendo el primero el cambio de la presidencia de Rafael Correa (2007-2017) a Lenin 
Moreno en el año 2017, evidencia un cambio en el modelo de gobierno, una izquierda menos radical, que como lo afirma el reconocido analista político Mauricio Jaramillo (2018) refiriéndose al giro político en Ecuador, "Ecuador es un caso aparte pero digamos que, dentro de las facciones de izquierda que dejó el expresidente Rafael Correa, la que prevalece es la más conservadora” (Jaramillo, 2018, citado en Quintana, 2018).

Con respecto a Chile, se destaca en el giro a la derecha la vuelta al poder del presidente conservador Sebastián Piñera (2017), en remplazo de la presidenta socialista Michelle Bachelet, quien había llegado al gobierno con una coalición de centroizquierda, esto dado al desencanto con las políticas del gobierno que lo sucede después de su primer gobierno (2010 y 2014).

En el ámbito de los países de Colombia y Perú, se puede afirmar que hay una profundización de la derecha. En Colombia el ascenso del presidente Iván Duque (2018-2022) en remplazo del presidente Francisco Santos (2010-2018) también de derecha, genera un cambio a una derecha mucho más fuerte, el cual es soportado por su partido Centro Democrático un partido conservador en alianza con los partidos más conservadores del país, a diferencia del anterior gobierno que recibió en su segundo gobierno el apoyo de partidos de izquierda.

En el Perú, se plantea también una continuidad de la política de derecha pero de mayor intensidad, tras el as- censo al gobierno de Martín Vizcarra en el año 2018, que como lo plantea Riesco (2018), Vizcarra es "un político independiente de ideas conservadoras y que fue escogido para concluir el mandato del empresario liberal Pedro Pablo Kuczynski, destituido por un escándalo de corrupción”.

Pero ¿qué generó ese giro a la derecha en Suramérica? Se puede afirmar que fueron varios aspectos tanto económicos como políticos, donde Riesco establece que:
"Según los analistas, la ola conser- vadora llegó a América Latina pre- cedida por una grave crisis econó- mica en varios de sus países provo- cada por la caída de los precios de las materias primas, principal pauta de exportación, y por los escánda- los de corrupción protagonizados por varios gobernantes de izquier- da. Con la crisis se erosionó el apo- yo que tuvieron los gobiernos de izquierda durante la bonanza eco- nómica” (Riesco, 2018, página 1).

Este panorama establece un giro a la derecha en especial en el Mercosur, a pesar de que Venezuela sigue inmersa en el Socialismo del siglo XXI, pero ha quedado sola y aislada, lo cual se refleja en varios aspectos, como la llegada del presidente Nicolás Maduro (2013-2019) con un menor respaldo popular que su antecesor y una notable pérdida de liderazgo en la región, y su reelección (2019-2025) muy cuestionada por su falta de respeto a la democracia, lo que determina su expulsión del bloque, cuya determinación del Mercosur establece "Suspender a 
la República Bolivariana de Venezuela en todos los derechos y obligaciones inherentes a su condición de Estado Parte del MERCOSUR, de conformidad con lo dispuesto en el segundo párrafo del artículo $5^{\circ}$ del Protocolo de Ushuaia. Implicaciones en la integración regional” (Mercosur, 2017).

Junto a Venezuela en la izquierda Suramericana está Bolivia, cuyo presidente Evo Morales presidente en el periodo 2005-2019, "se encamina a buscar una tercera reelección en el 2019, tras recibir luz verde del Tribunal Constitucional Plurinacional pese a que el año pasado le fue negada esa posibilidad en un referéndum" (BBC News Mundo, 2017).

Este panorama de gobiernos con giro a la derecha en la región, establece que "los latinoamericanos parecen mirar con mejores ojos al liberalismo económico que tanto cuestionaban tras las privatizaciones y dolorosas reformas de los años 90" (BBC News Mundo, 2017). Como lo afirman Jeifets, V. y Jeifets.L. (2016), "En Chile, Perú, Venezuela, Brasil las perspectivas del flanco de derecha están vinculadas, fundamentalmente, a los fracasos de la izquierda. Pero, ello no puede ser una garantía de éxito, pues será necesario para la derecha crear eficaces estructuras".

Pero hay que hacer alusión a no mirar el panorama en un extremismo de considerar que hay radicalismos en la región, a excepción del Brasil, se puede considerar que el giro a la derecha apunta más a colo- carse dentro de un ámbito político que podría denominarse más como conservador, que como lo manifiesta Jaramillo (2018), al afirmar que en la actualidad América Latina ya paso la página de la extrema radical y eso se hundió con la caída del proceso venezolano, y que el cambio que hay en la región es una tendencia hacia el conservatismo, el cual se mira en Chile, Argentina y Paraguay (Jaramillo 2018, citado en Quintana, 2018).

\section{La integración regional}

La región pasa de la izquierda a la derecha en la última década, ello implica una nueva visión sobre la integración regional suramericana, en donde los procesos de integración desarrollados por la ola de izquierda pueden sufrir problemas en su consolidación, dada la diferente dinámica generada por la nueva ola de derecha que imprime un nuevo derrotero en ella. En el ámbito de un "regionalismo abierto", que como lo plantea la CEPAL (1994), combina la integración económica y en gran medida el comercio en ámbitos de cercanía geográfica, que apunta a tendencia "natural" y no discriminatoria a terceros países; con una "integración impulsada por acuerdos o políticas explicitas, que sí entrañan ciertas preferencias con respecto al trato dispensado a las demás naciones" (CEPAL, 1994, p. 1).

Esta última orientación, responde a las prioridades del mercado en una visión de corto plazo, que prima lo más inmediato, la ampliación del mercado comercial. La posición de la CEPAL, 
desde un comienzo ha sido de apoyo a la política de regionalismo abierto, la cual responde en la economía latinoamericana como una respuesta a las crisis regionales y a las transformaciones en el panorama internacional, que para 1994, eran un lento avance de las negociaciones multilaterales, así como el avance de la comunidad económica europea y sin lugar a dudas el TLC Canadá - Estados Unidos -México, y lo que se resalta para este análisis, "la gradual convergencia de las políticas económicas aplicadas en América Latina y el Caribe y la creciente afinidad política entre gobiernos civiles y democráticamente electos" (CEPAL, 1994, p. 1).

Hoy en día el escenario no es diferente, todos los países están en democracia, han generado una ampliación de su comercio internacional a ámbitos latinoamericanos y tres economías suramericanas de corte liberal y con gobiernos de derecha tienen un marcado regionalismo abierto, con TLC con los Estados Unidos y con México, el referente Centroamericano. Sin lugar a dudas, el escenario dado por el giro a la derecha de las economías suramericanas vislumbra una mayor tolerancia de los gobiernos regionales al liberalismo comercial extra regional, en donde las economías más reacias a establecer vínculos con potencias económicas extranjeras como el Mercosur, hoy en día están terminando bajo la figura de Bloque a Bloque la negociación de un TLC con la Unión Europea, aspecto que a pesar de respetar la unidad de bloque comercial en la negociación, establece un comercio extra continental, el cual después de la negociación CAN-Mercosur (ACE 59 de 2005), es sin lugar a dudas el hecho más importante en la actividad comercial del Mercosur. Como lo reconoce el Mercosur, el proceso de negociación se ha extendido por espacio de 20 años y cobró un gran impulso en el año 2016, para ser firmado en el 2019 (Mercosur, 2019).

Este panorama de negociaciones que se podrían denominar "sur-norte" en la región suramericana, rompe la prioridad de la integración regional, dejándola en un segundo plano; el tamaño y la importancia del mercado externo sin lugar a dudas direccionan el fomento de la economía hacia dicho mercado. De manera individual las economías de la Alianza del Pacifico AP, apuntarán a su mercado norteamericano y las economías del Mercosur a su mercado europeo, es difícil de establecer que habrá un mercado común suramericano que aproveche independientemente la capacidad regional para sacar el mejor provecho de dichos acuerdos extra regionales, si el ámbito de dicho comercio de unos países con Norteamérica y otros con Europa se soporta en intereses particulares de mercados objetivos y sin lugar a dudas en el Mercosur de una clara política comercial no hegemónica, contrario a los países de la AP.

No obstante, como se ha podido ver a lo largo de los últimos años, existe la posibilidad de que los países con visiones más liberales, planteen la simultaneidad de políticas comer- 
ciales de profundizar la integración suramericana y a la vez mantener su integración extra regional, apuntando a una política de internacionalización de sus economías, que contrario con el ámbito de economías con un giro a la izquierda su rol es más comercial y económico que político. En contraste con lo que muchos creen, una visión contraria a la integración suramericana la tenía el gobierno de Hugo Chávez, quien en torno a las negociaciones CAN -Mercosur, "ha promovido una visión de Unasur como "alternativa" al modelo "neoliberal" que representaría tanto la CAN como Mercosur" (Sanahuja, 2009). Así mismo, en otro escenario de discusión en torno a la integración suramericana en el año 2006 en Cochabamba, el presidente Chávez manifestó que la CAN y el Mercosur no sirven (Sanahuja, 2009). Siendo su visión soportada en un regionalismo cerrado, anti hegemónico y de corte político.

Hoy en día tanto la OCDE como la CEPAL (2019) en relación a la problemática regional de bajo crecimiento de la actividad comercial, plantean que América Latina y el Caribe ALC, tienen grandes oportunidades para la integración regional, dado que en el año 2015 apenas $16 \%$ de las exportaciones totales de ALC se destinaron al mercado regional. Cifra baja comparada con la Unión Europea (63.2\%), el TLCAN (49.3\%) y la Asociación de Naciones del Sureste Asiático ASEAN (47\%). Como bien lo resaltan autores como Gudynas (2005), la CEPAL siempre ha tenido un sesgo de tipo comercial en torno a los acuerdos de integración, subordinando aspectos que según el autor serian claves como el laboral, el ambiental y el del manejo de fronteras, y también no se establecían mecanismos de coordinación política. Esto establece que la visión de dicha institución vea favorecido el ámbito liberal en la región pro comercio, pero también como lo plantean sus estudios, avanzar en elementos de una integración en ámbitos comunitarios, que posibiliten integrar el aparato productivo regional.

Contrario a lo que se pensaba que el gobierno de Brasil en cabeza del presidente Jair Bolsonaro, iba a tomar políticas proteccionistas y anti regionales, su posición ha sido impulsar el comercio regional, ello soportado en la gran importancia que tiene éste en sus exportaciones. Así mismo, su visión liberal se manifiesta en su claro respaldo a un regionalismo abierto, que como afirma Fiore (2019), "Pese a su supuesto compromiso con la región, tanto Bolsonaro como su ministro de Economía, Paulo Guedes, tienen entre sus objetivos dotar de mayor flexibilidad al Mercosur para que sus miembros puedan realizar acuerdos autónomos con otros países, evitando el bloque, lo cual aumentaría las exigencias de competitividad para las empresas argentinas y del resto de los países miembros" (p. 1).

Pero contradictorio a la flexibilidad dada por Brasil en el Mercosur, en beneficio de un ambiente regional abierto, que como se ha dicho no excluye a las economías con libre comercio extra regional, está la po- 
sición fuerte de Brasil y de las economías de derecha en Suramérica que zanjan posiciones políticas en el ámbito suramericano, tal es el caso de la conformación del Foro para el Progreso de América del Sur Prosur, en marzo del 2019, cuya reunión de los presidentes de Argentina, Brasil, Colombia, Ecuador, Paraguay y Perú; países con gobiernos de derecha, "tuvo como objetivo de ser la punta de lanza de un nuevo bloque para la integración regional que viene a reemplazar la Unasur" (Fiore, 2019 , p. 1). Este hecho muestra una clara intencionalidad política de este grupo de países en dicha institución, que no incluye a los países que tras la desintegración de la Unasur se mantienen activos como Bolivia, Uruguay, Venezuela, Guyana y Surinam. Siendo como lo afirma Fiore (2019), "el nuevo foro será un intento de institucionalizar el giro a la derecha que dio la región a partir de 2015”.

Si se aprecia por así decirlo el péndulo ideológico de la política en Suramérica en torno a las últimas décadas de derecha a izquierda, se puede afirmar que lo que ha llevado a tener un panorama suramericano de compromiso con la integración regional, han sido los puntos intermedios de esos polos, dado que la radicalización de posturas ha dispersado los intereses en torno a la región, por un lado la extrema derecha que ha sido hegemónica ve intereses fuera de la región, no apuntando a una mayor integración regional y en el mejor de los casos solo la comercial. No obstante, como pasa en la actualidad por el número de países en la derecha, su dinámica es mayor, así como también es un elemento de revanchismo con los procesos de integración generados por la izquierda cuando estaba frente al poder, es que se puede ver iniciativas como la de Prosur, no sin establecer su corte político e ideológico. Esto demuestra lo frágil de las instituciones creadas por la integración regional, en la medida que un cambio de los regímenes puede generar la pérdida de su vigencia y el esfuerzo y experiencia adquirida, sin dejar de reconocer otros ámbitos importantes dentro de dicha institucionalidad.

Así mismo lo anterior evidencia lo frágil del proceso integrador suramericano, al avanzar en niveles de mayor integración económica, sin una adecuada consolidación de las etapas, pues no es extraño escuchar que los bloques económicos en la región no han pasado de ser bloques comerciales limitados en comercio a comunidades imperfectas, que limitan en gran medida los objetivos planteados en su conformación, siendo este uno de los argumentos que se establecen al determinar el fracaso del proceso integrador en la región suramericana.

Por el lado de la izquierda, el aspecto anti ghegemónico del comercio regional ha generado el marginamiento de las economías al no tener una mayor participación de la integración regional, claro está también la divergencia política que incide en que un mayor interés de los países de derecha en dichos procesos regionales sea visto como un aval a su accionar político. 
En conclusión, se plantea que el regionalismo abierto entendido y como resultado de la voluntad de los países para insertarse en el mundo de la mejor manera, sea el ámbito donde confluyan los intereses nacionales, donde los intereses económicos y sociales primen por encima de los políticos, donde no haya injerencia de actores externos y esta decisión sea autónoma. Los países deben medir las potencialidades de corto y largo plazo en torno a su modelo de integración regional, cual es el que mejor les conviene.

Hoy en día se hace necesario el actuar como una región integrada económicamente hablando, que posibilite el beneficio de un gran mercado, un excelente proveedor de factores, un complemento de la actividad productiva, lo cual solo se puede hacer con una gran región, que como afirma la CEPAL (2017):

"El comercio intrarregional ofrece un gran potencial para las exportaciones de manufacturas y de productos de mayor elaboración en general, lo que pone de relieve la urgencia de profundizar la integración regional, más aún con el reciente giro de la política comercial de los Estados Unidos. ..,la suscripción de un acuerdo comercial de alcance regional traería aparejadas importantes ganancias, que serían mayores si tal acuerdo no se limitara a la reducción de aranceles sino que también incluyera la remoción de barreras no arancelarias y la armonización o reconocimiento mutuo de normas técnicas, sanitarias y fitosanitarias. En esta misma línea se incluye la adopción de mecanismos idóneos de acumulación de origen a nivel subregional y regional, con el propósito de impulsar la integración productiva y la formación de cadenas de valor regionales". (p. 71)

\section{Conclusiones}

Existe en la actualidad un giro a la derecha dado por la mayoría de las economías suramericanas, lo que establece que dichas economías buscaran los beneficios que otorgan los mercados internacionales y ello condiciona su accionar en torno a la integración regional, la cual está acorde a un regionalismo abierto, que no limita el ámbito económico de integración a entornos regionales sino también extra regionales, con un fuerte sesgo al ámbito comercial.

El gran peso tomado por la derecha en Suramérica por número de países y tamaño económico, genera una inclinación a una mayor integración comercial de la economía de la región, la cual se ve reflejado en la propuesta del Prosur como remplazo del Unasur, con la exclusión a las economías de izquierda, con excepción del Uruguay, que con una economía de corte progresista no accedió a participar en dicho grupo, argumentando el ámbito político marcado de dicho foro.

Hoy en día se puede afirmar que la integración suramericana no vislumbra una unidad, más bien se perfilan tres grupos de integración según sus gobiernos, uno inmerso en el regionalismo abierto y de corte hegemónico (Colombia, Perú y Chile), otros de re- 
gionalismo abierto dada su negociación con la Unión Europea (Brasil, Argentina, Paraguay y Uruguay) y por ultimo unos de regionalismo cerrado y anti hegemónico (Venezuela, Bolivia y en parte Ecuador, no obstante de pertenecer a Prosur y generar un proceso de transformación de su gobierno).

No cabe duda que lo que ha generado una fractura en los procesos de integración regional son los extremos en las posiciones políticas, que no establecen un clima adecuado para las negociaciones y la voluntad de integración, es de reconocer que el fracaso del ALCA generó un pegante en la región, con gobiernos interesados en la región, cuyo inicio del giro a la izquierda aglutinó a la mayoría de países, a tal punto que en sus inicios el Unasur tuvo la mayor participación de estos, pero con el paso del tiempo y las posiciones dadas por las profundizaciones del modelo generaron res- puestas de parte de los países tanto en el centro como en el otro extremo, a tal punto de su salida.

En este sentido se establece que lo ideal es generar un proyecto de integración regional estratégico que no pueda ser interferido por la política tan variable en la región, el cual aglutine los esfuerzos regionales en procura del mejor uso de sus recursos, como se ha planteado antes; una verdadera integración productiva con valor agregado, que posibilite ventajas en beneficio de la sociedad regional, donde el trabajo y la demanda interna de factores contribuyan a solventar los niveles de pobreza y desigualdad tan altos en la región, a los cuales los diagnósticos y pronósticos de las instituciones de mayor prestigio en la región apuestan con una integración regional a una mayor amplitud y profundidad, que ameritan avanzar a una integración más económica y no solo comercial. 


\section{Referencias}

Gil, J., \& Flórez, J. (2018). Brasil deja atrás 15 años de gobiernos de izquierda. El Colombiano, Publicado el 29 de octubre de 2018. Recuperado el 1 de julio de 2019 de https://www.elcolombiano.com/internacional/brasil-deja-atras-15anos-de-gobiernos-de-izquierda-DB9571162

Riesco, S. (2018). Latinoamérica se hace de derechas. Rtve. Recuperado 10 de julio de 2019 de http://www.rtve.es/noticias/20181030/latinoamerica-se-hace-derechas/1829180.shtml

Mercosur (2017). Suspensión de Venezuela en el Mercosur. Mercosur. Recuperado el 10 de julio de 2019 de https://www.mercosur.int/suspension-de-venezuela-en-el-mercosur/

BBC News Mundo (2017). Latinoamérica. ¿Está girando América Latina hacia la derecha?. El Comercio. Recuperado el 12 de julio de 2019 de https://elcomercio.pe/mundo/latinoamerica/dice-triunfo-sebastian-pinera-chile-giro-america-latina-derecha-noticia-482362-noticia/

Quintana, M. (2018). ¿Está América Latina dando un giro hacia la extrema derecha?. El Tiempo. - APP 28 de octubre 2018. Bogotá, Colombia. Recuperado el 10 de julio del 2019 de https://www.eltiempo.com/mundo/latinoamerica/ esta-america-latina-dando-un-giro-hacia-la-extrema-derecha-285796.

Jeifets, V., \& Jeifets, L. (2016). Resurgimiento de la Derecha. Particularidades y Perspectivas del Resurgimiento del "Fénix de la Derecha" en América Latina. En Iberoamérica, 3, 34-60.

Bobbio, N. (1996). Derecha e Izquierda. Razones y significados de una distinción política. Nueva edición revisada y ampliada. Madrid, España: Santillana, S.A. Taurus.

CEPAL (1994). El regionalismo abierto en América Latina y el Caribe: la integración económica al servicio de la transformación productiva con equidad. Comisión Económica Para América Latina y el Caribe. Santiago de Chile, 1994.

CEPAL (2017). Perspectivas del Comercio Internacional de América Latina y el Caribe, 2017 (LC/PUB.2017/22-P), Santiago, Chile.

Mercosur (2019). Acuerdo de Asociación Estratégica Mercosur-UE. Mercosur. Junio 2019. Recuperado el 15 de julio de 2019 de https://www.mercosur.int/ documento/mercosur-ue/_

Sanahuja, J. (2009). Del 'regionalismo abierto' al 'regionalismo post-liberal'. Crisis y cambio en la integración regional en América Latina y el Caribe. En: Anuario de la Integración de América Latina y el Gran Caribe 7, 11-54. Buenos 
Aires, Argentina. Recuperado el 1 de agosto del 2019 de https://www.researchgate.net/publication/303111093_Del_'regionalismo_abierto'_al_'regionalismo_postliberal'_Crisis_y_cambio_en_la_integracion_regional_en_America_Latina_y_el_Caribe_

OCDE et al. (2019). Perspectivas económicas de América Latina 2019: Desarrollo en transición, Paris: OECD Publishing. Recuperado el 30 de septiembre del 2019 de https://doi.org/10.1787/g2g9ff1a-es.

Gudynas, E. (2005). “El 'regionalismo abierto’ de la CEPAL: insuficiente y confuso," Programa de las Américas (Silver City, NM: International Relations Center, 28 de septiembre de 2005). Recuperado el 20 de julio del 2019 de http:/integracionsur.com/wpcontent/uploads/2016/10/GudynasRegionalismoAbiertoIrc.pdf

Fiore, G. (2019). Opinión. La integración regional en tiempos de la derecha. En: Comercio y Justicia. Recuperado el 19 de julio del 2019 de https:/comercioyjusticia.info/blog/opinion/la-integracion-regional-en-tiempos-de-la-derecha/ 